\title{
Estudos cinéticos da fotodegradação do corante alimentício amarelo tartrazina por processo oxidativo avançado
}

\section{Kinetics studies of photodegradation of tartrazine yellow food dye by advanced oxidative process}

DOI: $10.54019 /$ sesv2n3-003

\section{Karina Rodrigues de Fraga}

Graduanda em Licenciatura em Ciências exatas

Laboratório de Catálise, Universidade Federal do Rio Grande - FURG, Rua Barão do Caí, 125, CEP 95500-000, Santo Antônio da Patrulha, RS, Brasil. E-mail: fragak14@gmail.com

\section{Taís Port Hartz}

Engenheira Química, Mestranda no Programa em Química Tecnológica e Ambiental

Laboratório de Catálise, Universidade Federal do Rio Grande - FURG, Rua Barão do Caí, 125, CEP 95500-000, Santo Antônio da Patrulha, RS, Brasil. E-mail: taishartz@hotmail.com

\section{Carla Weber Scheeren}

Professora Doutora em Química da Escola de Química e Alimentos Laboratório de Catálise, Universidade Federal do Rio Grande - FURG, Rua Barão do Caí, 125, CEP 95500-000, Santo Antônio da Patrulha, RS, Brasil. E-mail: carlascheeren@gmail.com

\section{Karina Rodrigues de Fragaa}

Taís Port Hartza e Carla Weber Scheerena* aLaboratório de Catálise e Nanomateriais, Escola de Química e Alimentos, Universidade Federal do Rio Grande - FURG, Rua Barão do Caí, 125, CEP

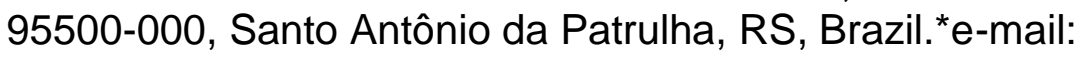

carlascheeren@gmail.com

\section{Resumo}

Neste estudo, o processo oxidativo avançado (POA) Foto-Fenton foi avaliado na degradação do corante alimentício amarelo tartrazina. A presença de corantes alimentícios em efluentes geram problemas ambientais devido à alta coloração e estabilidade dessas moléculas orgânicas, as quais são de difícil degradação e podem gerar subprodutos com alta toxicidade. O Processo Foto-Fenton apresenta 
eficiência na degradação de corantes, combinado as vantagens de simplicidade e baixo custo do processo. Foi estudado o POA Foto-Fenton [Ferro/peróxido de hidrogênio (H2O2)/luz ultravioleta (UV)]. Os parâmetros de tempo reacional, concentração e tempo de exposição a luz UV, foram avaliados para o corante amarelo tartrazina. Os resultados obtidos exibiram grande efetividade para o POA Foto-Fenton na degradação do corante amarelo tartrazina. O processo FotoFenton (Fe/H2O2) exibiu alta taxa de degradação do corante amarelo tartrazina em baixo tempo reacional. Os estudos cinéticos obtidos experimentalmente exibiram cinética de primeira ordem, com coeficiente de correlação $R 2=0,9786$ e constante $\mathrm{k}=1,079$.10-2 (L mol-1 min-1). Assim, podemos destacar que o POA estudado Foto-Fenton, destaca-s como um importante método para tratar efluentes industriais contendo corantes alimentícios, combinando as vantagens de facilidade, efetividade e baixo custo no processo.

Palavras-chave: POA; Corante alimentício; Amarelo Tartrazina, Degradação Cinética; Meio-ambiente.

\section{ABSTRACT}

In this study, the photo-Fenton advanced oxidative process (AOP) was evaluated in the degradation of the food dye tartrazine yellow. The presence of food dyes in effluents generates environmental problems due to the high coloration and stability of these organic molecules, which are difficult to degrade and can generate byproducts with high toxicity. The Photo-Fenton process presents efficiency in the degradation of dyes, combined the advantages of simplicity and low cost of the process. The Photo-Fenton POA [Iron/hydrogen peroxide (H2O2)/ultraviolet (UV) light] was studied. The parameters of reaction time, concentration and UV light exposure time were evaluated for the tartrazine yellow dye. The results obtained exhibited great effectiveness for the Photo-Fenton POA in the degradation of tartrazine yellow dye. The Photo-Fenton (Fe/H2O2) process exhibited high degradation rate of tartrazine yellow dye in low reaction time. The experimentally obtained kinetic studies exhibited first-order kinetics, with correlation coefficient $\mathrm{R} 2=0.9786$ and constant $\mathrm{k}=1.079 .10-2$ ( $\mathrm{L}$ mol-1 min-1). Thus, we can highlight that the studied Photo-Fenton POA, stands out as an important method to treat industrial effluents containing food dyes, combining the advantages of ease, effectiveness and low cost in the process.

Keywords: POA; Food Colorant; Tartrazine Yellow, Kinetic Degradation Environment.

\section{INTRODUÇÃO}

A presença de corantes, oriundos dos resíduos gerados pelas indústrias alimentícias, apresentam elevada concentração de matéria orgânica e forte coloração, sendo um dos grandes fatores de poluição em corpos d'água. A intensa coloração, os torna altamente detectáveis a olho nu, mesmo em concentrações tão baixas quanto $1 \mathrm{ppm}(1 \mathrm{mg} / \mathrm{L})$. Estes compostos apresentam grande estabilidade química, e seus subprodutos alta toxicidade. Entre os principais 
impactos causados, podemos destacar a eutrofização e as alterações na biota aquática, dificultando a passagem de luz solar e diminuindo o nível de oxigênio, consequentemente, reduzindo a atividade fotossintética (GUPTA; SUHAS, 2009; Andrade, Souza \& Couto, 1998; Nadais, Capela \& Arroja, 2005).

Os corantes alimentícios são compostos orgânicos utilizados principalmente para intensificar a coloração de alimentos e bebidas. Os principais corantes desta classe, são os orgânicos sintéticos artificiais, presentes em alimentos consumidos diariamente. Ao adquirirmos um produto alimentício, podemos constatar se ele foi colorido artificialmente através das informações descritas na embalagem.

Os métodos de tratamentos destes efluentes, buscam a efetiva redução e/ou remoção de contaminantes gerados no processo. (Crespilho \& Rezende, 2004; Feng, Zhu \& Hou, 2006). Os tratamentos físico-químicos de separação de fases (sedimentação, decantação, filtração, centrifugação, coagulação e flotação dos resíduos), destacam-se como métodos de tratamento clássico. Entretanto, existem processos alternativos como os Processos Oxidativos Avançados (POAs), que se baseiam na geração de espécies altamente oxidantes $(\mathrm{OH} \bullet)$, em quantidade suficiente para provocar a destruição dos poluentes, dentre eles a matéria orgânica (Nogueira \& Jardim, 1998; Crespilho, Santana \& Rezende, 2004; Nogueira \& Jardim, 1998; Pereira \& Freire, 2005; Tiburtius et al, 2005; Bertazzoli \& Pelegrini, 2002). Os POAs caracterizam-se como uma tecnologia limpa, aplicável ao tratamento de águas residuais, sendo necessária a adequação dos parâmetros experimentais ao tipo de efluente a ser tratado.

Diante dos fatos expostos, é importante destacar que os corantes têm grande importância econômica na indústria brasileira [por exemplo, em 20110 Brasil importou 158,4 mil toneladas (correspondente a US\$ 515 milhões) e exportou, no mesmo período, 62 mil toneladas (equivalente a US\$145 milhões) de corantes e pigmentos (ASSOCIAÇÃO BRASILEIRA DA INDÚSTRIA QUÍMICA, 2011]. Como exemplo, podemos citar o corante amarelo tartrazina, amplamente utilizado na indústria alimentícia, o qual pertence ao grupo funcional dos azocorantes e demonstra grande estabilidade frente ao calor e a luz solar. Dentre outros fatores, este corante também é citado em estudos, como contribuinte para 
problemas alérgicos graves e com potencial carcinogênico (Tripathi; Khanna; Das, 2007).

Neste contexto, o presente trabalho de pesquisa buscou aplicar o POA Foto-Fenton na efetiva degradação do corante alimentício amarelo tartrazina, combinando o uso de ferro, peróxido de hidrogênio e luz ultravioleta.

Na tabela 1 estão expostas as características, aplicações, efeitos causados e países onde o uso do corante amarelo tartrazina é proibido.

Tabela 1. Características, aplicações e proibições dos Corante alimentício amarelo tartrazina

Corante Aplicações na Indústria Alimentícia Efeitos causados Proibições em Países

Amarelo Tartrazina Tinta do alcatrão de carvão usado em laticínios, licores, fermentados, produtos de cereais, frutas, iogurtes. Reações alérgicas (asma, bronquite, rinite, náusea, broncoespasmo, urticária, eczema, dor de cabeça, eosinofilia) e inibição da agregação plaquetária à semelhança dos salicilatos. Insônia em crianças associada à falta de concentração e impulsividade. Reação alérgica cruzada com salicilatos (ácido acetilsalisílico). Pode provocar hiperatividade em crianças quando associado ao benzoato de sódio. Seu uso deve ser indicado nos rótulos no Brasil, nos EUA e na Inglaterra.

\section{MATERIAL E MÉTODOS}

\subsection{Estrutura Química Do Corante Alimentício Estudado Amarelo}

Tartrazina.

Corante Estrutura Química

Amarelo Tartrazina

\section{2 metodologia}

Para a realização dos experimentos foi preparada uma solução aquosa do corante alimentício Amarelo Tartrazina, dissolvendo-se 0,1g do respectivo corante em $200 \mathrm{~mL}$ de água. A solução aquosa do corante foi posteriormente submetida ao tratamento Foto-Fenton. O processo Foto-Fenton, foi realizado utilizando-se palha de aço (Ferro), peróxido de Hidrogênio (H2O2, 36 \% v/v) e luz Ultravioleta (UV). Para a realização dos experimentos foi adicionado $4,0 \mathrm{~g}$ de palha de aço (Ferro) combinado com 10 gotas de Peróxido de hidrogênio (H2O2, 36 \% v/v) e exposição a luz UV. Os experimentos foram realizados utilizando-se um fotoreator composto de um sistema de agitação, termômetro digital e uma fonte de luz 
UV (constituída de uma lâmpada de vapor de mercúrio de baixa pressão de 150 W, da qual removeu-se o bulbo). As soluções foram submetidas a agitação (para homogeneização da solução) e alíquotas da solução do corante amarelo tartrazina foram retiradas para análise por espectrofotometria UV-visível, nos intervalos de 30 a 150 min. Em outro experimento, uma solução foi mantida em repouso e a outra foi mantida sob agitação e exposição à luz ultravioleta, para avaliar o efeito da agitação no processo.

As imagens das soluções do corante amarelo tartrazina foram registradas, nos intervalos de 30 a 150 minutos ao longo do POA Foto-Fenton, para avaliar as mudanças na coloração da solução do corante amarelo tartrazina, assim como, as análises de absorbância por espectrofotometria UV-visível.

\subsection{Análise por Espectrofotometria UV-visível}

A degradação da solução do corante amarelo tartrazina pelo processo FotoFenton foi acompanhada por espectrofotometria UV-visível (Perkin Elmer Lambda 25 UV/Vis Spectrometer) na faixa de comprimento de onda de 200 a $600 \mathrm{~nm}$. Todos os ensaios foram realizados em triplicata (Ribani et al, 2004). As medições de absorbância das soluções contendo o corante amarelo tartrazina foram realizadas nos intervalos de 30,60, 90, 120, e 150.

\section{RESULTADOS E DISCUSSÃO}

Os resultados obtidos na degradação da solução do corante amarelo tartrazina pelo processo Foto-Fenton, estão expostos em relação a remoção da coloração da solução (monitorada por Espectrometria UV-Vis). Com base nos resultados obtidos no desenvolvimento deste trabalho, pode-se destacar a alta eficiência do processo Foto-Fenton aplicado na degradação do corante amarelo tartrazina.

O processo Foto-Fenton combina o uso de palha de Aço (Ferro), peróxido de hidrogênio e exposição a luz UV. O processo sem agitação apresentou mudança na coloração apenas após 72 horas de tratamento, enquanto, a solução com palha de Aço combinado com peróxido de hidrogênio e luz UV sob agitação exibiu redução na coloração da solução após 30 minutos de tratamento FotoFenton.

$\mathrm{Na}$ Tabela 2 estão expostos os dados de diminuição da concentração da solução do corante amarelo tartrazina submetido ao tratamento por POA FotoFenton com agitação, nos intervalos de tempo de análise 0, 30, 60, 90, 120 e 150 minutos (após análise por espectrofotometria UV-visível). 
Tabela 2. Tempo de tratamento e concentração da solução do corante amarelo tartrazina submetida ao processo Foto-Fenton

Tempo de tratamento $(\min ) \quad$ Concentração do corante Amarelo Tartrazina na solução (mg L-1)

$\begin{array}{ll}0 & 0,47 \\ 30 & 0,34 \\ 60 & 0,24 \\ 90 & 0,16 \\ 120 & 0,10 \\ 150 & 0,03\end{array}$

Na Figura 1 está exposto o gráfico de absorbância obtidos por espectrofotometria UV-visível nos intervalos de tempo de 0, 30, 60, 90, 120 e 150 minutos de degradação da solução do corante amarelo tartrazina submetido ao tratamento pelo processo Foto-fenton.

Figura 1. Espectro UV/Vis do corante alimentício amarelo tartrazina submetido ao tratamento FotoFenton, em diferentes tempos de degradação

Na Figura 1 está exposto o espectro de absorbância da solução contendo o corante amarelo tartrazina durante o processo de degradação pelo processo Foto-Fenton por 150 minutos. No espectro é possível observar as bandas de absorção característica do corante amarelo tartrazina $(L=428 \mathrm{~nm})$.

A banda de absorção em regiões próximas a $260 \mathrm{~nm}$ indica a presença de carbono orgânico aromático (Souza \& Daniel, 2005). A degradação das soluções dos corantes pode ser comprovada pelo decréscimo das bandas características ao longo do tempo.

Com base na análise da redução de absorbância durante o processo de degradação fotoquímico, observa-se que houve uma redução de $99 \%$ da coloração da solução contendo o corante amarelo tartrazina após 150 minutos de tratamento sob agitação (Figura 2).

Figura 2. Imagens da remoção da coloração da solução do corante amarelo tartrazina com o Processo Foto-Fenton: (1a) tempo 30; (1b) tempo $60 \mathrm{~min}$; (1c) tempo $90 \mathrm{~min}$; (1d) tempo $120 \mathrm{~min}$; (1e) tempo $150 \mathrm{~min}$.

Na Figura 3 pode-se verificar o decréscimo da concentração do corante no decorrer do tempo de degradação sem agitação, sendo possível observar que somente após 72 horas a solução apresenta início da descoloração da solução. Após 144 horas de reação sem agitação a solução está incolor. Este resultado expõe a importância da agitação constante do sistema no processo Foto-Fenton para a obtenção de melhores resultados de degradação do corante alimentício amarelo tartrazina. 
Figura 3. Imagens da remoção da coloração da solução do corante amarelo tartrazina com o Processo Foto-Fenton sem agitação: (1a) tempo zero (1b) tempo 72 horas; (1c) tempo 144 estudos horas.

A partir da análise da Figura 4, observa-se a diminuição da absorbância da solução do corante alimentício amarelo tartrazina em função do tempo e que, a partir de 150 minutos, há uma tendência de estabilização do sistema. A partir dos dados de redução da absorbância durante o processo de degradação pelo processo Foto-fenton, foi possível observar uma redução de 99\% na cor das soluções contendo os corante amarelo tartrazina (Figura 4).

Figura 4. Curva de degradação do corante alimentício amarelo tartrazina pelo processo FotoFenton em função do tempo.

A Figura 5 exibe os resultados da avaliação da diminuição da absorção da solução do corante alimentício amarelo tartrazina em função do tempo em minutos (Abst/Abs0 versus tempo), onde o Abs0 representa absorvância no instante $t=0$, enquanto o Abst indica absorbância no instante arbitrário t.

A equação da diminuição da absorbância em função do tempo obtida para o corante alimentício amarelo tartrazina foi $y=1,1116 e-0,015 x$, com $R 2=0,9182$.

Figura 5. Curva de degradação do corante alimentício amarelo tartrazina pelo processo FotoFenton

A cinética de degradação que ocorre na redução de compostos orgânicos em solução aquosa pode ser de primeira ordem ou ordem zero, estando relacionado se é baixa ou alta a sua concentração. Se há linearidade do gráfico de concentração em função do tempo, então a cinética é de ordem zero.

Na cinética de primeira ordem, o logaritmo natural da concentração dos reagentes deve diminuir linearmente com o tempo (Atkins, 1988). Usualmente o limiar de concentração (Bolton et al, 2001) alta ou baixa é 100 mg.L-1.

A avaliação da cinética de degradação foi realizada a partir da construção de um gráfico In (Abst/Abs0) em função do tempo (Atkins, 1988). A Figura 6 mostra os dados obtidos experimentalmente, e uma cinética de primeira ordem pode ser observada. Os dados obtidos com a degradação do corante alimentício amarelo tartrazina estão de acordo com dados expostos na literatura (Araújo, Yokoyama \& Teixeira, 2006; Bolton et al, 2001; Catanho, Malpass \& Motheo, 2006).

$\mathrm{Na}$ cinética de primeira ordem, o logaritmo natural da concentração de reagentes deve diminuir linearmente ao longo do tempo (Atkins, 1988). Normalmente o limiar de concentração (Bolton et al, 2001) alto ou baixo é de 100mg. L-1. 
Na Figura 6 é exibida a equação linear de primeira-ordem (linha de tendência linear) para o corante alimentício amarelo tartrazina, $y=-0,0134 x+0,1527$, com coeficiente de correlação $\mathrm{R} 2=0,9786$.

Figura 6. Cinética de primeira ordem obtida para a degradação do corante alimentício amarelo tartrazina.

O valor da constante de descoloração $(\mathrm{k})$, usando a equação para reações de primeira-ordem $(\ln [\mathrm{A}]-\ln [\mathrm{A}] 0=-\mathrm{kt})$, da reação de degradação (Atkins, 1988) foi igual a $\mathrm{k}=1,079.10-2(\mathrm{~L} \mathrm{~mol}-1$ min-1). O resultado obtido com a degradação do corante amarelo tartrazina é concordante com dados obtidos pela literatura (Araújo, Yokoyama \& Teixeira, 2006; Bolton et al, 2001; Catanho, Malpass \& Motheo, 2006).

\section{Adequação à Legislação Ambiental}

Com base nos resultados obtidos neste trabalho, com níveis de descarte permitidos por resoluções e normativas (Ministério do Meio Ambiente) pode-se destacar que, para corantes artificiais, estes devem estar virtualmente ausentes (Resolução CONAMA № 357/2005); o efluente não deve conferir mudança acentuada de coloração ao corpo receptor no ponto de lançamento.

\section{CONCLUSÕES}

Os resultados obtidos nesta pesquisa exibiram alta eficiência do POA FotoFenton na degradação do corante alimentício amarelo tartrazina, de amplo uso na indústria alimentícia. A aplicação do tratamento Foto-Fenton (Fe/H2O2/UV) mostrou redução de $99 \%$ na cor e concentração das soluções do corante estudado. O processo Foto-Fenton gerou um resultado de degradação mais eficiente sob agitação da solução, em um intervalo de tempo de reação curto, exibindo mudanças na cor e concentração de soluções do corante; destaca-se que após a filtragem, foram obtidas soluções incolores. O estudo cinético foi analisado e os dados obtidos experimentalmente demonstraram cinética de primeira ordem, com coeficiente de correlação $\mathrm{R} 2=0,9786$, e a constante de descoloração $(k)$ da reação de degradação com um valor de $k=1,079.10-2$ ( $L$ mol-1 min-1) para o corante alimentício amarelo tartazina. 


\section{REFERÊNCIAS}

1.Montoia P, Água, o "Ouro" Azul do Nosso Século. (2006), http://didaktos.ua.pt/recursos/didaktos, 4828.pdf.

2. Heng JZX, Tang, KY, Regulacio MD, Lin M, Loh XJ, Li Z, Ye E (2021) Solar-Powered Photodegradation of Pollutant Dyes Using Silver-Embedded Porous TiO2 Nanofibers. Nanomaterials, 11, 856.

3. Andrade RCB, Souza MFL, Couto, ECG (1998) Influência de Efluentes Têxteis e Alimentícios sobre o Metabolismo e Propriedades Físicas e Químicas do Rio Piauitinga (Sergipe), Química Nova, 21, 424 - 427.

4. Nadais H, Capela I, Arroja L (2005) Treatment of Dairy Wastewater in UASB Reactors Inoculated with Flocculent Biomass. Water SA, 31, 603 - 608

5. Gupta VK, Suhas (2009) Application of low-cost adsorbents for dye removal a review. Journal of Environmental Management, 90, 2313-2342.

6.Crespilho FN, Rezende MOO (2004) Eletro flotação: Princípios e Aplicações, Brasil, 1 a ed, 77.

7. Feng X, Zhu S, Hou H (2006) Investigation of 207 Nm UV Radiation for Degradation of Organic Dye in Water. Water S. A, 32, $43-48$.

8. Zhu H, Jiang R, Xiao L, et al (2009) Photocatalytic decolorization and degradation of Congo red on innovative crosslinked chitosan/nano-CdS composite catalyst under visible light irradiation. Journal of Hazardous Materials, 169 (1-3), 933-940.

9.De Sá FP, et al (2020) Fotodescoloração dos corantes alimentícios vermelho eritrosina e azul brilhante por fotólise direta. Brazilian Journal of Development, Curitiba, 6, 7, 46707-46718.

10.Sökmen M, Allen DW, Akkaş F, et al (2001) Photo-Degradation of Some Dyes using Ag-Loaded Titaniumdioxide. Water, Air, \& Soil Pollution, 132, 153-163.

11.Da Silva CP, Marmitt S, Haetinger C, Stulp S (2008) Avaliação da degradação do corante vermelho bordeaux através de processo fotoquímico. Eng. Sanit. Ambient., 13 (1), 73-77.

12.Alshabanat MN, Al-Anaz MM (2018) An Experimental Study of Photocatalytic Degradation of Congo Red Using Polymer Nanocomposite Films, Hindawi Journal of Chemistry. ID 9651850, 1-8.

13.Associação Brasileira da Indústria Química. Corantes. (2011). http://www.abiquim.org.br

14.Tripathi M, Khanna SK, Das M (2007) Surveillance on use of synthetic colours in eatables vis a vis prevention of food adulteration act of India. Food Control, 18, 211-219. 
15.Ribani M, et al. (2004) Validação em métodos cromatográficos e eletroforéticos. Química Nova, 27, 771 - 780.

16. Souza JB, Daniel LA (2005) Comparação entre Hipoclorito de Sódio e Ácido Peracético na Inativação de E. Coli, Colifagos E C. Perfringens em Água com Elevada Concentração de Matéria Orgânica. Engenharia Sanitária e Ambiental, 10, 111-117.

17.Resolução Conama № 357, 17 de março 2005 (cetesb.sp.gov.br). 18.Aquino RVS, Barbosa AA, Ribeiro LB, et al (2019) Degradation of leaf green food dye by heterogeneous photocatalysis with $\mathrm{TiO} 2$ over a polyethylene terephthalate plate. Chem. Pap. 73, 2501-2512.

19.Zazouli MA, Ghanbari F, Yousef IM, Madihi-Bidgoli S (2017) Photocatalytic degradation of food dye by $\mathrm{Fe} 3 \mathrm{O} 4-\mathrm{TiO} 2$ nanoparticles in presence of peroxymonosulfate: The effect of UV sources, Journal of Environmental Chemical Engineering, 5(3), 2459-2468.

20.Shanker U, Rani M, Jassal V (2017) Degradation of hazardous organic dyes in water by nanomaterials. Environ Chem Lett, 15, 623-642.

21.Nalliah RE (2015) Oxone/Fe2+ Degradation of Food Dyes: Demonstration of Catalyst-Like Behavior and Kinetic Separation of Color. J. Chem. Educ. 92, 10, 1681-1683.T 